\title{
Personalised treatment in the epilepsies: challenges and opportunities
}

\begin{abstract}
Introduction. 'Personalised' medicine is not a new construct. Indeed, the science of medicine has been historically focused around notions of subgroups and categorization. A few decades ago the Human Genome Project provided a complete resource of detailed information about the structure, organization, and function of the full set of human genes. This has enabled implementation of the idea that treatments could be targeted to genetically-defined subgroups of individuals.

Areas covered. We provide an overview of the current pitfalls and opportunities of 'personalised' treatment in epilepsy. Development of targeted therapeutics has been so far mainly by re-purposing drugs already available on the market but not necessarily approved for the treatment of epilepsy. Most genomic findings have not yet translated into widespread utilisation for therapy.

Expert opinion/Commentary. Genotypic and phenotypic heterogeneity, epigenetic modulation, variable penetrance and expressivity, environmental influence, post-translational changes are all factors that increase the complexity of genomic networks and generate pitfalls in the application of the 'personalised' treatment paradigm. Unravelling the multiple layers of biological complexity through new collaborative and computational approaches, and deep and multimodal phenotyping, is becoming fundamental for a better understanding of disease biology and in turn better tailored treatment strategies.
\end{abstract}

Keywords: epilepsy, genomic, personalised medicine, translational medicine, genetic heterogeneity, phenotypic heterogeneity 


\section{Introduction}

In 1990, the first association of a genetic mutation with a specific form of epilepsy was reported [1]. Since then the epilepsy-associated genome has greatly expanded, and a genetic contribution to epilepsy causation is estimated to be present in about $70 \%$ of people with epilepsy [2]. In particular, the advent of next-generation sequencing technologies has enabled high-throughput gene identification studies and allowed a molecular diagnosis in about $25 \%$ of patients with Mendelian disorders [3]. The identification of the underlying causal gene variants is crucial for the application of the currently popular 'precision medicine' paradigm, according to which disease treatment and prevention can be tailored to the individual variability in genes, environment, and lifestyle for each person [4]. This concept was in fact already suggested by Hippocrates, when stating that 'human beings are innately (genetically) different from one another, and this individuality affects both their predisposition/susceptibility to disease and their response to therapeutics', recognising therefore the central principle of precision medicine [5]. Hippocrates suggested that treatment should be causeoriented rather than symptom-oriented, highlighting the importance of understanding disease pathophysiology in order to formulate a rational, targeted therapeutic strategy [6]. Unfortunately, after thousands of years we have barely begun to dissect epilepsy pathophysiology and although the list of single genes linked to epilepsy is expanding rapidly, the analysis and interpretation of the large number of variants generated by next-generation sequencing studies remains challenging. Reduced penetrance, variable expressivity (which must have an element of genetic contribution in themselves) phenocopies, and genetic heterogeneity are common issues in diagnostic assessment of Mendelian epilepsy syndromes. Despite notable research advances in epilepsy genomics, a translational gap still exists, and personalized treatment approaches are available only in a minority of genetic epilepsies, with most genomic findings not yet translated into widespread utilisation in the clinic. Here we discuss the current main pitfalls and complexity of 'personalised' treatment approach in epilepsy and provide an overview of possible future directions in epilepsy genomics.

\section{The genome, and genetic heterogeneity}

In humans there are roughly 21,000 protein-coding genes, and genome-wide variation from one person to another can be up to $0.5 \%$ of the entire genome [7]. One of the world's largest collaborative biological efforts, the Human Genome Project, was completed in 2003, and gave us the first complete sequence of the human genome. Since then the search for genes implicated in common human diseases has accelerated significantly. However, gene mutations may not always result in abnormal protein function or in phenotypic expression. This can be explained by the integration of intrinsic and environmental signals in the genome that can modulate gene expression at various levels, including DNA transcription, alternative RNA splicing, translation, and posttranslational protein modifications [8,9]. For example, enhancers can modulate gene transcription, and subsequently misregulate gene expression in disease [10,11]; histone modifications affect gene expression and have a fundamental role in most biological processes [12]. In epilepsy so far genetic research has mostly focused on individual genes or proteins related to certain phenotypes. It is now becoming clearer that variable expressivity and penetrance due to gene-gene and gene-environment interactions should be taken into account, in order to fully understand disease biology and therefore be able to apply the precision medicine concept.

Genetic heterogeneity is a recurrent feature of clinical epilepsy syndromes. There are many examples of this, such as the well-known Dravet syndrome, where in most cases, the cause is a mutation in the voltage- gated sodium channel type I alpha subunit gene, SCN1A [13], and it represents by far the most frequent indication for DNA analysis within the group of epileptic encephalopathies. However, the yield of $S C N 1 A$ mutations is around $85 \%$, implying that other genes or SCN1A-related factors may be involved in the same phenotype, such as the PCDH19 gene [14]. Another factor explaining ' $S C N 1 A$-negative' Dravet syndrome is somatic mosaicism, with a minimal prevalence of SCN1A mosaic deletion recently estimated at $0.9 \%$ (95\% confidence level: 
0.11-3.11\%) [15]. Previously incomplete genome annotation may also cause missed diagnoses in patients with epilepsy; therefore iterative interrogation of clinical exome sequence data that were initially found to be inconclusive, in particular with re-evaluation of well-defined alternative exons in known epilepsy genes, is needed when there are negative exome results [16]. Familial nocturnal frontal lobe epilepsy is a further example of genetic heterogeneity, with mutations in the nicotinic acetylcholine receptor subunit genes CHRNA4 and CHRNB2 responsible for the clinical phenotype in only about $12-15 \%$ of cases of autosomal dominant nocturnal frontal lobe epilepsy [17]. Only a single mutation has been described in CHRNA2, encoding the alpha2 subunit of the nicotinic acetylcholine receptor, in a large family of Italian origin with nocturnal frontal lobe epilepsy with atypical features such as wandering and ictal fear [18]. Other genes have also been associated with familial nocturnal frontal lobe epilepsy, including KCNT1 [19] and DEPDC5 [20].

Genetic heterogeneity is also derived from the high diversity of molecular pathways underlying epilepsy, likely mostly converging into impact on inhibitory processes [21]. These pathways include every aspect of synaptic inhibitory transmission from early development through to maturation of adult GABA neurotransmission, with the resulting inhibitory defects underlying epilepsy phenotypes.

A further element to add to the complexity of genetics in the epilepsies is the detection of deleterious mutations in epilepsy genes, in particular voltage and ligand-gated ion channel genes, in people without epilepsy, and the detection of more than one mutation in known human epilepsy genes in people with epilepsy [22]. All these findings show the probable complex allelic architecture underlying the epilepsy phenotype in each individual. Epistasis, where the effect of a locus on a trait is conditional on genotypes observed at other loci, may therefore become fundamental for personalised treatment approaches, requiring that the full profile of variants present in an individual be considered before estimating the clinical impact of any single one [23]. A parallel sequencing study of 237 channel genes in people with epilepsy and unaffected controls showed that structural variants in both known and suspected epilepsy genes were present also in controls, implying that even putatively deleterious ion channel mutations confer uncertain epilepsy risk to an individual depending on the interaction with the full profile of variants [22]. Modifier genes associated with epilepsy and determining epistatic interaction have been demonstrated in mouse models [24-26].

Novel and effective tools leading to biological understanding of such multivariant complexity are required for personalised medicine strategies: these include computation modeling of biological networks; deep genotype-phenotype correlations in large cohorts of people with epilepsy with novel tools; and standardised functional characterisation and validation of gene mutations, for example, in neurons derived from induced pluripotent stem cells (IPSCs) from the affected individual [27].

\section{The phenome, and heterogeneity of phenotype}

The rapid development of high-throughput sequencing technologies has led to a growing list of genes with causative mutations in many epilepsy syndromes [28]. However, sometimes the identification of novel causative genes is currently mostly first based on exome or genome sequencing studies and then requires detailed assessment of the phenotype to better understand the phenotype-genotype association. Historically, phenotyping was the guiding element to gene identification, as for example the careful distinction between nocturnal frontal lobe seizures and parasomnias was a key component to identify mutations of the CHRNA4 gene as a cause of autosomal dominant nocturnal frontal lobe epilepsy [29]. As more and more genes with causal de novo mutations for rare epilepsy syndromes with Mendelian inheritance are identified, the need grows for collaborative studies designed to dissect the phenotype-genotype correlation for a given gene, and the spectrum of disease is often expanded as extreme phenotypes not previously reported emerge. However, 'extended' phenotypes might also be explained by multilocus genomic variation 
and two Mendelian diseases can coexist in the same patient, with 'distinct' or 'overlapping' phenotypes [30]. The situation becomes even more complex for common epilepsies with complex genetic architecture [31], where one of the factors impeding gene discovery is the lack of homogeneous subgroups of phenotypes big enough to provide reasonable power to detect causal genetic variants. A very interesting study recently used a novel approach to reconcile the discrepancy between detailed phenotyping and large-scale genetic studies of epilepsies [32]. They used the Epilepsy Phenome/genome Project (EPGP) dataset and examined familial aggregation of focal seizure types and seizure symptoms among relatives with non-acquired focal epilepsies. They found familial aggregation of specific seizure semiology and seizure types within non-acquired focal epilepsies, showing that systematic phenotyping can reveal phenotypic aggregations that are beyond our current understanding of the underlying genetic framework. This also suggests how the phenome may still be guiding genetic studies, especially when there is 'complex inheritance', with multiple variants and environmental factors contributing to the cause, none of which having a major effect on disease risk when acting by itself [33]. Overall, phenotype can be determined by 'distinct' and 'shared' genetic influence, with different implications in risk of inheritance and concordance of epilepsy phenotype in families with multiple affected individuals $[33,34]$.

Variable expressivity, when the same gene, allele or mutation in a single gene can produce different epilepsy phenotypes in different individuals, typically in a family, is also likely due to modifying effects of other genes or environmental factors. For example, in a family with generalized epilepsy with febrile seizures plus (GEFS+), a single mutation in SCN1A was associated with a broad phenotypic spectrum including typical febrile seizures, febrile seizures plus (i.e., febrile seizures persisting beyond age six, or accompanied by afebrile generalized tonic seizures), idiopathic generalized epilepsy, temporal lobe epilepsy, myoclonic-astatic epilepsy, or Dravet syndrome [35]. Pleiotropy [36] is particularly prevalent in genetic channelopathies; this might be explained by various factors, including the presence of mutational hotspots [37], specific functional effects of single mutations (i.e., the recurrent $K C N Q 2$ p.Arg198Glyn variant has been found in cases presenting with a peculiar phenotype and was shown to cause gain-of-function in vitro; [38]), and presence of genetic modifiers $[39,40]$.

\section{Current 'personalised' approaches in genetic epilepsies}

'Personalised' treatment in epilepsy aims to target the underlying molecular dysfunction and in turn reverse the neuroelectrophysiological and neurochemical deficits that may lead to seizures and associated neurological dysfunction [41]. So far, the subset of genetic epilepsies where 'personalised' treatment approaches are available form a minority, but confirm that the proof of principle exists and hopefully will be gradually extended to most epilepsy cases where a molecular dysfunction is identified.

GLUT-1 deficiency syndrome is a genetic metabolic encephalopathy due to mutations in the $S L C 2 A 1$ gene, which encodes the glucose type I transporter (GLUT-1), resulting in a defect in glucose transport into the central nervous system. GLUT-1 deficiency shows wide phenotypic pleiotropy, including including early onset epileptic encephalopathy, paroxysmal exercise-induced dyskinesia, and early childhood-onset refractory absence epilepsy. The gold standard treatment is the ketogenic diet, which bypasses the defective glucose transport and provides an alternative energy supply to the brain [42]. Early 'precision' diagnosis and treatment in this syndrome are crucial to provide brain nourishment and control seizures [43].

Pyridoxine (vitamin B6)-dependent epilepsy is caused by bi-allelic mutations in the ALDH7A1 gene, which encodes antiquitin. Deficiency of antiquitin causes seizures because accumulating 1piperideine-6-carboxylate (P6C) condenses with pyridoxal 5-phosphate (PLP) and inactivates this latter enzyme cofactor essential for normal metabolism of neurotransmitters. ALDH7Al analysis could also be used for prenatal diagnosis of pyridoxine-dependent epilepsy. Seizures are often fully controlled by treatment with pyridoxine [44]. B6-responsive seizures may also be due to mutations 
in the pyridox(am)ine 5'-phosphate oxidase $(P N P O)$ gene, and in some cases may be better treated with pyridoxal 5'-phosphate [45]. A lysine-restricted diet, the aim of which is to restrict formation of potentially toxic intermediate metabolites, has been recently proposed as an adjunctive treatment in cases where response to pyridoxine is incomplete, although the evidence for benefit remains limited [46,47].

Tuberous sclerosis complex (TSC) is an autosomal dominant, multi-organ disease with widely variable clinical expression, caused by heterozygous germline mutations in the tumor suppressor genes TSC1 and TSC2 [48]. Protein products of these genes, hamartin and tuberin, have been shown to form a heterodimer (TSC1-TSC2 complex) that inhibits the mechanistic target of rapamycin (mTOR) signaling cascade [49]. The mTOR signaling cascade is a key homeostatic regulatory pathway involved in cell growth and cell replication. It is hypothesised that lack of tuberin (or a functional tuberin-hamartin complex) results in loss of GTPase activity, and inappropriate or constitutive activation of the ras superfamily of proteins, thereby removing an inhibitory influence on the cell cycle. The clinical manifestations of TSC are quite distinctive and include hamartomatous lesions of the brain, skin, heart, lungs and kidneys, in addition to a wide spectrum of neurological features, including seizures, autism, and intellectual disability [50]. The tumours in TSC develop following inactivation of both alleles of either TSC1 or TSC2 loci, resulting in a loss of control of cell growth and cell division, consistent with the "two-hit" Knudson tumour suppressor gene hypothesis [51]. mTOR inhibitors such as everolimus may help to reverse the molecular defect associated with TSC, and ameliorate the clinical consequences of the disorder [52]. Recently a phase three, randomized, double-blind, placebo controlled study (EXIST-3) showed that everolimus is effective for treatment of epilepsy in patients with tuberous sclerosis, independent of the presence of sub-ependymal giant cell astrocytomas [53]. Other genes are involved in the mTOR pathways and mutations in those genes have been associated with focal epilepsies (e.g. DEPDC5, NPRL2, NPRL3 and MTOR itself); treatment with mTOR inhibitors might represent a 'precision' treatment also in these cases, but multi-centre randomised clinical trials are needed.

The gene $K C N T 1$ encodes a sodium-dependent potassium channel and is activated by increased intracellular chloride and sodium concentrations; it is responsible for the slow hyperpolarization of the transmembrane potential during action potentials [54]. KCNT1 gain-of-function mutations are reported to cause early-onset epileptic encephalopathies including epilepsy of infancy with migrating focal seizures (EIFMS). In vitro testing has indicated that the electrophysiological defect of at least some of these mutations may be reversed by quinidine, an antiarrhythmic drug, which is a partial blocker of KCNT1[55]. In three cases of epilepsy of infancy with migrating seizures due to $K C N T 1$ mutations, quinidine resulted in decreased seizure frequency or freedom from seizures and improved psychomotor development [56-58]. However, another two cases with early-onset epileptic encephalopathy associated with KCNT1 mutations manifesting gain-of-function in vitro, one showing a novel phenotype with developmental regression and severe nocturnal focal and secondarily generalised seizures starting in early childhood, and the other with early-onset epileptic encephalopathy $[57,59]$ did not respond to treatment with quinidine. The current evidence suggests that quinidine represent a promising candidate for a precision medicine approach in some KNCT1related epilepsy syndromes, but further studies in larger cohorts of patients are necessary to clarify its effectiveness.

The $K C N Q 2$ and $K C N Q 3$ genes encode subunits of the voltage-gated potassium $\mathrm{M}$ channel underlying the neuronal M-current [60], and are amongst the most common causes of neonatal epileptic encephalopathy of widely varying severity [61]. In $K C N Q 2 / K C N Q 3$-related epilepsy there is a potential tailored precision medicine strategy with the use of retigabine (ezogabine), a drug primarily acting as a positive allosteric modulator of KCNQ2-5 (Kv7.2-7.5) ion channels, and the first neuronal potassium $(\mathrm{K}+)$ channel opener licensed for the treatment of epilepsy [62]. In vitro studies identified the probable binding site of retigabine in KCNQ2 and KCNQ3 channels, explaining its voltage-dependent activating effect through a hyperpolarizing shift of the activation 
curve [63]. Retigabine has been shown to partially reverse the effect of $K C N Q 2$ mutations in cell models [64].

Preliminary data from humans with $K C N Q 2$-related disease suggest that retigabine may be a useful treatment option with improvement of seizure frequency and development in 3 of 4 patients treated before the age of 6 months whereas treatment at an older age was less successful ( 2 of 7 patients) [65]. However, mainly due to the discovery of additional side effects on long-term treatment, including skin and retinal pigmentation potentially leading to visual loss, the production of retigabine has been discontinued. Also, it has been hypothesised that it may not be equally effective for all functional subgroups of these conditions, as detrimental clinical effects were observed in a few patients with specific gain-of-function variants [66]. Sodium channel blockers also seem effective in $K C N Q 2$-related epilepsy [67], possibly because voltage-gated sodium channels and KCNQ potassium channels co-localize and may form a channel complex whose excitability could be modified by sodium channel blockers [68]. Sodium channel blockers including carbamazepine and phenytoin should be considered as first-line treatment in patients with $K C N Q 2$-related epilepsy, as there is a suggestion that early effective treatment reduces cognitive disability [69]. There are other examples of 'personalised' approaches in epilepsy, including the avoidance of sodium channel blockers in patients with Dravet syndrome [70], the avoidance of sodium valproate in patients with epilepsy related to $P O L G$ mutations [71], the use of sodium channel blockers in patients with $S C N 2 A$ - and $S C N 8 A$-related epilepsies with gain-of-function mutations [72,73], and the potential use of memantine in patients with epileptic encephalopathy due to GRIN2A mutations [74].

In summary, current 'personalised' treatment strategies include drugs with an effect on ion channels (either openers or blockers), on the mTOR pathway, or on brain metabolism by providing an alternative energy supply in order to bypass defective transport mechanisms. So far, drugs or treatments that have been investigated were those already available on the market but not necessarily approved for the treatment of epilepsy (i.e., re-purposed) (summary in Table 1 and Figure 1).

'Personalised' treatment should also take into account the genetic variation that can influence response to antiepileptic treatment through pharmacokinetic mechanisms. Polymorphism in genes encoding drug metabolizing enzymes, mostly belonging to the cytochrome P450 (CYP) family, may affect serum concentrations of antiepileptic drugs, or alter flux through paths for drug metabolism, with subsequent potential risk of drug toxicity. For example, there is established evidence of an effect of polymorphic $C Y P 2 C 9$ and $C Y P 2 C 19$ genes, where allelic variation can lead to significant differences in serum concentrations of antiepileptic drugs [75]. CYP2C9 accounts for about $90 \%$ of the metabolism of phenytoin. Individuals carrying CYP2C9 alleles encoding variant enzymes (allozymes) with reduced activity show a slower rate of phenytoin metabolism compared with individuals homozygous for the wild-type allele, and therefore have a greater risk of developing concentration-dependent neurotoxicity [76]. CYP2C19 polymorphisms influence the rate of phenobarbital metabolism, with ethnic differences in its tolerability profile [77]. However, despite the available evidence, pre-treatment pharmacogenetic testing is not routine practice, and the standard approach remains the monitoring of clinical signs of toxicity and drug levels [78].

\section{Genetic complexity}

Although the molecular diagnosis of an early-onset epilepsy syndrome can have an important impact on therapeutic management and subsequently bring about a dramatic change in disease outcome, immediate clinical translation of genetic discoveries is not often possible. As discussed above, genetic and phenotypic heterogeneity certainly contribute to the complexity of genotypephenotype associations. Many open questions remain, and various approaches should be considered to address this complexity. 
Causation in the epilepsies is increasingly being attributed to rare variation, as in the epileptic encephalopathies [79] and familial epilepsies [80]. A recent case-control sequencing study of exome data from patients with familial genetic generalised epilepsy, or familial or sporadic nonacquired focal epilepsy, the two most common epilepsy syndromes, revealed a significant excess of ultra-rare deleterious variation in known epileptic encephalopathy genes [81], showing how, as in the rare epilepsies, genetic risk arises in a minority of cases from ultra-rare variants with large effect including de novo mutations. This would imply that the precision medicine paradigm could be applied also in the more common epilepsies. However, it remains unclear why common epilepsies are generally less severe than epileptic encephalopathies and how these findings can be translated into clinical practice.

An important study on rare variants in the GluN2A and GluN2B (encoded by GRIN2A and GRIN2B) subunits of the $N$-methyl- $D$-aspartate receptor (NMDAR), showed various mechanisms leading to NMDAR dysfunction [37]. These include alterations to agonist binding, channel gating, receptor biogenesis, and forward trafficking. This work is a clear example of the complexity of genotype-phenotype (molecular and clinical) correlation, and shows how both gain- and loss-of-function variants in the same gene can be associated with similar neurologic disorders.

In any individual, disease mechanisms should be ideally considered by integrating the effect of a given genetic mutation with the thousands of both common and rare inherited variants, that together interact in a genomic network, with additional layers of epigenetic and other modulation. This will be challenging. Also, the intrinsic impact of a variant at the single cell level may vary depending on 'its molecular neighbourhood' [82]. Further layers of integration emerge when interpreting complex phenotypes such as behaviour or cognition. Epigenetic mechanisms, including DNA methylation and post-translational histone modifications, are involved in driving experience-dependent gene expression underlying memory formation [83]. Altered promoter DNA methylation profiles have been described in a number of genes associated with seizures or epilepsy in vitro and in vivo, including BRD2 [84], CPA6 [85], Gria2 [86], Grin2b [87], and RELN [88]. Genome-wide alteration of DNA methylation has been described as a general pathomechanism associated with epileptogenesis and epilepsy in experimental animal models, although there seems to be a limited overlap betwen different models [89]. Furthermore, the dynamic regulation of DNA methylation by neuronal activity [90] may have implications in the epileptogenic process.

Post-translational modification of histones includes acetylation, methylation and phosphorylation. Altered histone acetylation due to changes in the expression of histone deacetylases has been shown to have a significant role in epileptogenesis [91]. Histone methylation at lysine and arginine residues can mediate both transcriptional repression and activation, depending on the target site, and the association of epilepsy with genetic defects involving histone methyltransferases suggests a role of the enzymes catalysing the methylation reaction in epileptogenesis [92]. Increase in phosphorylation of histone $\mathrm{H} 3$ has been reported after pilocarpine- and kainate-induced seizures or status epilepticus in mice [93,94]. Epitranscriptomics, which includes activity-induced RNA modifications, RNA editing, dynamic changes in the secondary structure of RNA, and RNA localization, seems to have a crucial role in brain experience-dependent plasticity and adaptive behaviour [95].

\section{Taming complexity: the role of deep phenotyping}

We are probably at the beginning of a new era of 'personalised' treatment in epilepsy. As discussed above, there are many exciting opportunities, but also significant challenges. Probably, the identification of a presumably causal genetic variant represents just the first step in understanding the biological dysfunction leading to epilepsy and subsequently formulating an individual 'precision' treatment. Deep phenotype characterisation may become very important in better 
understanding the origin and the mechanisms underlying the spectrum of clinical variability in epilepsy, and in better characterising the disease in an individual.

Phenotypes provided by further, novel, modalities of investigation (beyond clinical assessment, neuroimaging and neurophysiological studies) might strengthen the correlation with genetic patterns, and help interpret genomic complexity. For example, specific analysis of 3D face images, based on 3D stereophotogrammetry and Dense Surface Modelling (DSM), a powerful and objective method of detecting face shape abnormalities, can be useful in identifying atypical face shape in adults or children with genomic structural variants [96,97]. Transcranial Magnetic Stimulation (TMS), which can be used non-invasively to stimulate the cerebral cortex, allows exploration of cortical physiology by measuring neuronal excitability indirectly by electromyography recordings (TMS-EMG) following motor cortex stimulation, or by simultaneous EEG recording (TMS-EEG), with direct probing of cortical excitability even in non-motor areas. By applying TMS-EMG in two monogenic conditions we demonstrated meaningful profiles of systems neurophysiology. We found impaired GABA Aergic intracortical neurotransmission in SCN1A mutation-bearing Dravet Syndrome [98], confirming in humans the "interneurone hypothesis", according to which SCN1A mutation results in reduced function of GABAergic inhibitory interneurons, leading to an overall excessive neuronal excitation. This is currently the best-supported pathophysiological explanation of Dravet Syndrome [99,100]. However, the pathophysiology of Dravet Syndrome has not yet been entirely elucidated, and for example, other studies using patient-derived neurons have suggested increased excitability of both GABAergic and glutamatergic neurons [27].

In another genetically-homogeneous condition, alternating hemiplegia of childhood, we found symptomatic and asymptomatic fluctuations in motor cortex excitability [101], not seen in controls, suggesting that instability of excitability underlies hemiplegic attacks, linking altered function of mutant ATP1A3 to clinical manifestation, and suggesting that stabilisation of membrane excitability might be a therapeutic option. These represent examples of 'precision physiology', where deep phenotypic analysis may provide a better understanding of the system biology, possibly including the supramolecular mechanisms bridging single gene defects to neurophysiological dysfunction of brain circuits [82]. Indeed, neurophysiological studies enable correlation of the dysfunction at the cellular level with alterations at the brain dynamics level, and demonstration of how local perturbations associated with susceptibility or disease-conferring variants converge into pathogenetic pathways and finally culminate in abnormal phenotypes.

Transcriptomic single cell profiling has emerged as a powerful approach to characterization and classification of neurons [102]. The potential causal relationships between transcriptomic signatures and specific morphological, physiological and functional properties remain to be elucidated [103]. Furthemore, extending the phenotypic analysis to organs than the brain, ideally at the individualwide scale, is also relevant for 'personalised' treatment, as some genetic defects affect multiple organs, and broad phenotyping can inform diagnostic and therapeutic management [104].

\section{Conclusion}

'Personalised' treatment approaches are currently available only in a minority of genetic epilepsies, with most genomic findings not yet translated into widespread utilisation in the clinic. There is a high level of complexity underlying genotype-phenotype correlation, and only a full understanding of the disease biology can lead to successful tailored treatment. Novel pathophysiological studies, including integrated analyses of neuroimaging, neurophysiology, genetics and epigenetics, will contribute to deep and multimodal phenotypic characterisation that is fundamental for the functional characterisation of the genotype.

\section{Expert commentary}


The key weakness in the 'personalised' treatment approach in epilepsy is the lack of full understanding of the underlying disease mechanism. Even when the epilepsy is caused by a single gene deleterious mutation with Mendelian inheritance, multiple supramolecular mechanisms may affect its phenotypic presentation and in turn influence the therapeutic response. Identification of underlying mechanisms of epilepsy raise the potential to reuse already-licensed drugs, and there are already examples of successful drug repurposing for the treatment of drugresistant epilepsies [55,74]. However, most current 'personalised' approaches in epilepsy using repurposed drugs derive from anecdotal observations in individual patients and need to be confirmed in randomised controlled clinical trials. Other 'personalised' treatment modalities include: the use of potential antiepileptic compounds with specific molecular targets on traditional test platforms, such as ion channel openers or blockers, i.e. retigabine [65]; the use of natural products such as cannabidiol with anticonvulsive effects in animal models but also effective in epileptic encephalopathies as shown in recent clinical trials [105]; the use of diet intervention specifically targeting GLUT1 deficiency syndrome providing an energy source for the brain independent of the defective glucose transporter [42]; the use of compounds modulating the mTOR pathway such as everolimus and in turn reversing some of the neurological conditions associated with gene mutations in this pathway [52]; the targeting of long non-coding RNA to upregulate haploinsufficient gene expression in mouse models of Dravet syndrome [106]; and the use of stem cell-based therapeutic approaches in mouse models [107].

These all represent promising modalities of intervention and some have already been applied in humans with successful outcomes. However, open questions and challenges remain. So far, most genetic defects have been modelled in animals and of course the translation to humans might be affected by differences in gross brain structure, regional organisation and gene expression. In this review, we emphasize the role of deep phenotyping as one of the key elements to fill the gap between genetic models and human disease. We show how multimodal characterisation of the phenotype could reveal disease mechanisms and enables deeper understanding of system biology in humans. Integration of deep phenotyping with in vitro and in vivo models of genetic defects may lead to greater understanding of the neurophysiological dysfunction underlying the epilepsy, and may expedite the delivery of phenotype-based treatment to each individual with epilepsy.

\section{Five-year view}

Focused studies on the functional biology of human gene mutations are essential to the success of precision medicine. New collaborative and computational approaches are required to uncover, validate, and simulate genetic variants in complex combinations. Deep, multimodal and broad phenotyping is fundamental to analysis of the correlation with the genomic layout, and to extrapolate the role of further layers of complexity. At present, caution is still required in the application of personalised medicine strategies in epilepsy. Further advances are needed to fully link genomic information to disease expression and course, and optimize the selection of therapeutic targets in each individual. In this, genomics is a tool, one component of the overall picture. Basic, clinical, and translational approaches should be closely integrated to drive the development of precision treatment, and so significantly transform clinical care in epilepsy.

\section{Key issues}

- Despite notable research advances in epilepsy genomics, a translational gap still exists, and personalized treatment approaches are available only in a minority of genetic epilepsies, with most genomic findings not yet translated into widespread utilisation in the clinic. 
- The concept of 'personalised' treatment is not new. The rapid development of highthroughput sequencing technologies has led to a growing list of genes with causative mutations in many epilepsy syndromes, and has enabled implementation of the idea that treatments could be targeted to genetically-defined subgroups of individuals.

- The analysis and interpretation of the large number of variants generated by next-generation sequencing studies remains challenging.

- Genotypic and phenotypic heterogeneity, epigenetic modulation, variable penetrance and expressivity, environmental influence, post-translational changes are all factors that increase the complexity of genomic networks and generate pitfalls in the application of the 'personalised' treatment paradigm.

- Phenotypes provided by further, novel, modalities of investigation (beyond clinical assessment, neuroimaging and neurophysiological studies) might strengthen the correlation with genetic patterns, and help interpret genomic complexity.

- Development of targeted therapeutics in epilepsy has been so far mainly by repurposing drugs already available on the market; other potential strategies exist.

- At present, caution is still required in the application of the personalised medicine strategy in epilepsy. Further advances are needed to fully link genomic information to the disease expression and course, and optimize the selection of therapeutic targets in each individual.

\section{Figure legend}

Figure 1. Summary of current 'personalised' treatment strategies and their targets at the cellular level. These include drugs with an effect on ion channels (either openers or blockers), on brain metabolism by providing an alternative energy supply in order to bypass defective transport mechanisms (i.e. ketogenic diet), or mTOR pathway inhibitors (i.e., rapamycin or everolimus). 


\section{References}

1. Shoffner JM, Lott MT, Lezza AM, et al. Myoclonic epilepsy and ragged-red fiber disease (MERRF) is associated with a mitochondrial DNA tRNA(Lys) mutation. Cell 1990; 61:931-937

2. Hardies K, Weckhuysen S, De Jonghe P, et al. Lessons learned from gene identification studies in Mendelian epilepsy disorders. Eur. J. Hum. Genet. 2016; 24:961-967

3. Yang Y, Muzny DM, Xia F, et al. Molecular findings among patients referred for clinical wholeexome sequencing. JAMA 2014; 312:1870-1879

4. National Research Council (US) Committee on A Framework for Developing a New Taxonomy of Disease. Toward Precision Medicine: Building a Knowledge Network for Biomedical Research and a New Taxonomy of Disease. 2011

5. Sykiotis GP, Kalliolias GD, Papavassiliou AG. Pharmacogenetic principles in the Hippocratic writings. J Clin Pharmacol 2005; 45:1218-1220

6. Hippocrates. Hippocrates. Loeb Classical Library, 1931.

7. Roberts R, Wells GA, Stewart AFR, et al. The genome-wide association study--a new era for common polygenic disorders. J Cardiovasc Transl Res 2010; 3:173-182

8. Martinez NJ, Walhout AJM. The interplay between transcription factors and microRNAs in genome-scale regulatory networks. Bioessays 2009; 31:435-445

9. Adeli K. Translational control mechanisms in metabolic regulation: critical role of RNA binding proteins, microRNAs, and cytoplasmic RNA granules. Am. J. Physiol. Endocrinol. Metab. 2011; 301:E1051-64

10. Maurano MT, Humbert R, Rynes E, et al. Systematic localization of common disease-associated variation in regulatory DNA. Science 2012; 337:1190-1195

11. Plank JL, Dean A. Enhancer function: mechanistic and genome-wide insights come together. Mol. Cell 2014; 55:5-14

12. Bannister AJ, Kouzarides T. Regulation of chromatin by histone modifications. Cell Res. 2011; 21:381-395

13. Zuberi SM, Brunklaus A, Birch R, et al. Genotype-phenotype associations in SCN1A-related epilepsies. Neurology 2011; 76:594-600

14. Djémié T, Weckhuysen S, Spiczak von S, et al. Pitfalls in genetic testing: the story of missed SCN1A mutations. Mol Genet Genomic Med 2016; 4:457-464

15. Nakayama T, Ishii A, Yoshida T, et al. Somatic mosaic deletions involving SCN1A cause Dravet syndrome. Am. J. Med. Genet. A 2018; 12:197-662

16. Epilepsy Genetics Initiative. De novo variants in the alternative exon 5 of SCN8A cause 
epileptic encephalopathy. Genet. Med. 2017; 57:1027

17. Steinlein OK. Genetic heterogeneity in familial nocturnal frontal lobe epilepsy. Prog. Brain Res. $2014 ; 213: 1-15$

18. Aridon P, Marini C, Di Resta C, et al. Increased sensitivity of the neuronal nicotinic receptor alpha 2 subunit causes familial epilepsy with nocturnal wandering and ictal fear. Am. J. Hum. Genet. 2006; 79:342-350

19. Heron SE, Smith KR, Bahlo M, et al. Missense mutations in the sodium-gated potassium channel gene KCNT1 cause severe autosomal dominant nocturnal frontal lobe epilepsy. Nat. Genet. 2012; 44:1188-1190

20. Picard F, Makrythanasis P, Navarro V, et al. DEPDC5 mutations in families presenting as autosomal dominant nocturnal frontal lobe epilepsy. Neurology 2014; 82:2101-2106

21. Noebels J. Pathway-driven discovery of epilepsy genes. Nat. Neurosci. 2015; 18:344-350

22. Klassen T, Davis C, Goldman A, et al. Exome sequencing of ion channel genes reveals complex profiles confounding personal risk assessment in epilepsy. Cell 2011; 145:1036-1048

** Parallel exome sequencing study in epilepsy patients and unaffected controls showed high level of complexity of the allelic architecture underlying personal disease risk of epilepsy, demonstrating that deleterious ion channel mutations can be present also in unaffected individuals.

23. Holmes GL, Noebels JL. The Epilepsy Spectrum: Targeting Future Research Challenges. Cold Spring Harb Perspect Med 2016; 6:a028043

24. Kearney JA, Yang Y, Beyer B, et al. Severe epilepsy resulting from genetic interaction between Scn2a and Kenq2. Hum. Mol. Genet. 2006; 15:1043-1048

25. Glasscock E, Qian J, Yoo JW, et al. Masking epilepsy by combining two epilepsy genes. Nat. Neurosci. 2007; 10:1554-1558

26. Calhoun JD, Hawkins NA, Zachwieja NJ, et al. Cacna1g is a genetic modifier of epilepsy in a mouse model of Dravet syndrome. Epilepsia 2017; 58:e111-e115

27. Liu Y, Lopez-Santiago LF, Yuan Y, et al. Dravet syndrome patient-derived neurons suggest a novel epilepsy mechanism. Ann. Neurol. 2013; 74:128-139

28. Epi4K Consortium, Epilepsy Phenome/Genome Project, Allen AS, et al. De novo mutations in epileptic encephalopathies. Nature 2013; 501:217-221

* Exome sequencing study of 264 trios including probands with epileptic encephalopathies, focusing on genes exceptionally intolerant to sequence variation. Substantial interconnections with autism and intellectual disability gene networks were found.

29. Steinlein OK, Mulley JC, Propping P, et al. A missense mutation in the neuronal nicotinic acetylcholine receptor alpha 4 subunit is associated with autosomal dominant nocturnal frontal lobe epilepsy. Nat. Genet. 1995; 11:201-203

30. Posey JE, Harel T, Liu P, et al. Resolution of Disease Phenotypes Resulting from Multilocus 
Genomic Variation. N. Engl. J. Med. 2017; 376:21-31

31. Sisodiya SM, Mefford HC. Genetic contribution to common epilepsies. Curr. Opin. Neurol. $2011 ; 24: 140-145$

32. Tobochnik S, Fahlstrom R, Shain C, et al. Familial aggregation of focal seizure semiology in the Epilepsy Phenome/Genome Project. Neurology 2017; 89:22-28

33. Ottman R. Analysis of genetically complex epilepsies. Epilepsia 2005; 46 Suppl 10:7-14

34. Peljto AL, Barker-Cummings C, Vasoli VM, et al. Familial risk of epilepsy: a population-based study. Brain 2014; 137:795-805

35. Mulley JC, Scheffer IE, Petrou S, et al. Channelopathies as a genetic cause of epilepsy. Curr. Opin. Neurol. 2003; 16:171-176

36. Paaby AB, Rockman MV. The many faces of pleiotropy. Trends Genet. 2013; 29:66-73

37. Swanger SA, Chen W, Wells G, et al. Mechanistic Insight into NMDA Receptor Dysregulation by Rare Variants in the GluN2A and GluN2B Agonist Binding Domains. Am. J. Hum. Genet. 2016; 99:1261-1280

38. Millichap JJ, Miceli F, De Maria M, et al. Infantile spasms and encephalopathy without preceding neonatal seizures caused by KCNQ2 R198Q, a gain-of-function variant. Epilepsia 2017; 58:e10-e15

39. Calhoun JD, Hawkins NA, Zachwieja NJ, et al. Cacna1 gis a genetic modifier of epilepsy caused by mutation of voltage-gated sodium channel Scn2a. Epilepsia 2016; 57:e103-e107

40. Kapplinger JD, Erickson A, Asuri S, et al. KCNQ1 p.L353L affects splicing and modifies the phenotype in a founder population with long QT syndrome type 1. J. Med. Genet. 2017; 54:390398

41. EpiPM Consortium. A roadmap for precision medicine in the epilepsies. Lancet Neurol 2015; $14: 1219-1228$

42. De Vivo DC, Leary L, Wang D. Glucose transporter 1 deficiency syndrome and other glycolytic defects. J. Child Neurol. 2002; 17 Suppl 3:3S15-23-discussion 3S24-5

43. Pong AW, Geary BR, Engelstad KM, et al. Glucose transporter type I deficiency syndrome: epilepsy phenotypes and outcomes. Epilepsia 2012; 53:1503-1510

44. Mills PB, Struys E, Jakobs C, et al. Mutations in antiquitin in individuals with pyridoxinedependent seizures. Nat. Med. 2006; 12:307-309

45. Mills PB, Camuzeaux SSM, Footitt EJ, et al. Epilepsy due to PNPO mutations: genotype, environment and treatment affect presentation and outcome. Brain 2014; 137:1350-1360

46. van Karnebeek CDM, Stockler-Ipsiroglu S, Jaggumantri S, et al. Lysine-Restricted Diet as Adjunct Therapy for Pyridoxine-Dependent Epilepsy: The PDE Consortium Consensus

Recommendations. JIMD Rep 2014; 15:1-11 
47. Teneiji Al A, Bruun TUJ, Cordeiro D, et al. Phenotype, biochemical features, genotype and treatment outcome of pyridoxine-dependent epilepsy. Metab Brain Dis 2017; 32:443-451

48. Curatolo P, Bombardieri R, Jozwiak S. Tuberous sclerosis. Lancet 2008; 372:657-668

49. Kwiatkowski DJ, Manning BD. Tuberous sclerosis: a GAP at the crossroads of multiple signaling pathways. Hum. Mol. Genet. 2005; 14 Spec No. 2:R251-8

50. Henske EP, Jozwiak S, Kingswood JC, et al. Tuberous sclerosis complex. Nat Rev Dis Primers 2016; $2: 16035$

51. Henske EP, Neumann HP, Scheithauer BW, et al. Loss of heterozygosity in the tuberous sclerosis (TSC2) region of chromosome band 16p13 occurs in sporadic as well as TSC-associated renal angiomyolipomas. Genes Chromosomes Cancer 1995; 13:295-298

52. Citraro R, Leo A, Constanti A, et al. mTOR pathway inhibition as a new therapeutic strategy in epilepsy and epileptogenesis. Pharmacol. Res. 2016; 107:333-343

53. French JA, Lawson JA, Yapici Z, et al. Adjunctive everolimus therapy for treatment-resistant focal-onset seizures associated with tuberous sclerosis (EXIST-3): a phase 3, randomised, doubleblind, placebo-controlled study. Lancet 2016; 388:2153-2163

* Phase 3, randomised, double-blind, placebo-controlled study, showing that adjunctive everolimus treatment significantly reduced seizure frequency with a tolerable safety profile compared with placebo in patients with tuberous sclerosis complex and treatment-resistant seizures.

54. Barcia G, Fleming MR, Deligniere A, et al. De novo gain-of-function KCNT1 channel mutations cause malignant migrating partial seizures of infancy. Nat. Genet. 2012; 44:1255-1259

55. Milligan CJ, Li M, Gazina EV, et al. KCNT1 gain of function in 2 epilepsy phenotypes is reversed by quinidine. Ann. Neurol. 2014; 75:581-590

56. Bearden D, Strong A, Ehnot J, et al. Targeted treatment of migrating partial seizures of infancy with quinidine. Ann. Neurol. 2014; 76:457-461

57. Mikati MA, Jiang Y-H, Carboni M, et al. Quinidine in the treatment of KCNT1-positive epilepsies. Ann. Neurol. 2015; 78:995-999

58. Zhang Q, Li J, Zhao Y, et al. Gene mutation analysis of 175 Chinese patients with early-onset epileptic encephalopathy. Clin. Genet. 2017; 91:717-724

59. Chong PF, Nakamura R, Saitsu H, et al. Ineffective quinidine therapy in early onset epileptic encephalopathy with KCNT1 mutation. Ann. Neurol. 2016; 79:502-503

60. Wang HS, Pan Z, Shi W, et al. KCNQ2 and KCNQ3 potassium channel subunits: molecular correlates of the M-channel. Science 1998; 282:1890-1893

61. Shellhaas RA, Wusthoff CJ, Tsuchida TN, et al. Profile of neonatal epilepsies: Characteristics of a prospective US cohort. Neurology 2017; 89:893-899

62. Sachdeo R, Partiot A, Biton V, et al. A novel design for a dose finding, safety, and drug 
interaction study of an antiepileptic drug (retigabine) in early clinical development. Int J Clin Pharmacol Ther 2014; 52:509-518

63. Schenzer A, Friedrich T, Pusch M, et al. Molecular determinants of KCNQ (Kv7) K+ channel sensitivity to the anticonvulsant retigabine. J. Neurosci. 2005; 25:5051-5060

64. Wang J, Li Y, Hui Z, et al. Functional analysis of potassium channels in Kv7.2 G271V mutant causing early onset familial epilepsy. Brain Res. 2015; 1616:112-122

65. Millichap JJ, Park KL, Tsuchida T, et al. KCNQ2 encephalopathy: Features, mutational hot spots, and ezogabine treatment of 11 patients. Neurol Genet 2016; 2:e96

66. Mulkey SB, Ben-Zeev B, Nicolai J, et al. Neonatal nonepileptic myoclonus is a prominent clinical feature of KCNQ2 gain-of-function variants R201C and R201H. Epilepsia 2017; 58:436445

67. Numis AL, Angriman M, Sullivan JE, et al. KCNQ2 encephalopathy: delineation of the electroclinical phenotype and treatment response. Neurology 2014; 82:368-370

68. Pan Z, Kao T, Horvath Z, et al. A common ankyrin-G-based mechanism retains KCNQ and $\mathrm{NaV}$ channels at electrically active domains of the axon. J. Neurosci. 2006; 26:2599-2613

69. Pisano T, Numis AL, Heavin SB, et al. Early and effective treatment of KCNQ2 encephalopathy. Epilepsia 2015; 56:685-691

70. Genton P. When antiepileptic drugs aggravate epilepsy. Brain Dev. 2000; 22:75-80

71. Li S, Guo J, Ying Z, et al. Valproic acid-induced hepatotoxicity in Alpers syndrome is associated with mitochondrial permeability transition pore opening-dependent apoptotic sensitivity in an induced pluripotent stem cell model. Hepatology 2015; 61:1730-1739

72. Wolff M, Johannesen KM, Hedrich UBS, et al. Genetic and phenotypic heterogeneity suggest therapeutic implications in SCN2A-related disorders. Brain 2017; 140:1316-1336.

73. Barker BS, Ottolini M, Wagnon JL, et al. The SCN8A encephalopathy mutation p.Ile1327Val displays elevated sensitivity to the anticonvulsant phenytoin. Epilepsia 2016; 57:1458-1466

74. Pierson TM, Yuan H, Marsh ED, et al. GRIN2A mutation and early-onset epileptic encephalopathy: personalized therapy with memantine. Ann Clin Transl Neurol 2014; 1:190-198

75. Lopez-Garcia MA, Feria-Romero IA, Fernando-Serrano H, et al. Genetic polymorphisms associated with antiepileptic metabolism. Front Biosci (Elite Ed) 2014; 6:377-386

76. Depondt C, Godard P, Espel RS, et al. A candidate gene study of antiepileptic drug tolerability and efficacy identifies an association of CYP2C9 variants with phenytoin toxicity. Eur. J. Neurol. 2011; 18:1159-1164

77. Goto S, Seo T, Murata T, et al. Population estimation of the effects of cytochrome P450 2C9 and 2C19 polymorphisms on phenobarbital clearance in Japanese. Ther Drug Monit 2007; 29:118121 
78. Balestrini S, Sisodiya SM. Pharmacogenomics in epilepsy. Neurosci. Lett. 2017;

79. McTague A, Howell KB, Cross JH, et al. The genetic landscape of the epileptic encephalopathies of infancy and childhood. Lancet Neurol 2016; 15:304-316

80. Baulac S, Ishida S, Marsan E, et al. Familial focal epilepsy with focal cortical dysplasia due to DEPDC5 mutations. Ann. Neurol. 2015; 77:675-683

81. Epi4K Consortium, Epilepsy Phenome/Genome Project. Ultra-rare genetic variation in common epilepsies: a case-control sequencing study. Lancet Neurol 2017; 16:135-143

* Case-control exome sequencing study showing excess of ultra-rare variation in known epilepsy gene in two common epilepsy syndromes: familial genetic generalised epilepsy, or familial or sporadic non-acquired focal epilepsy, suggesting that targeting of treatments to the genetic cause in rare devastating epilepsies might also extend to a proportion of common epilepsies

82. Noebels J. Precision physiology and rescue of brain ion channel disorders. J. Gen. Physiol. 2017; 149:533-546

83. Lopez-Atalaya JP, Barco A. Can changes in histone acetylation contribute to memory formation? Trends Genet. 2014; 30:529-539

84. Pathak S, Miller J, Morris EC, et al. DNA methylation of the BRD2 promoter is associated with juvenile myoclonic epilepsy in Caucasians. Epilepsia 2018; 59:1011-1019

85. Belhedi N, Perroud N, Karege F, et al. Increased CPA6 promoter methylation in focal epilepsy and in febrile seizures. Epilepsy Res. 2014; 108:144-148

86. Machnes ZM, Huang TCT, Chang PKY, et al. DNA methylation mediates persistent epileptiform activity in vitro and in vivo. PLoS ONE 2013; 8:e76299

87. Ryley Parrish R, Albertson AJ, Buckingham SC, et al. Status epilepticus triggers early and late alterations in brain-derived neurotrophic factor and NMDA glutamate receptor Grin2b DNA methylation levels in the hippocampus. Neuroscience 2013; 248:602-619

88. Kobow K, Jeske I, Hildebrandt M, et al. Increased reelin promoter methylation is associated with granule cell dispersion in human temporal lobe epilepsy. J. Neuropathol. Exp. Neurol. 2009; 68:356-364

89. Dębski KJ, Pitkanen A, Puhakka N, et al. Etiology matters - Genomic DNA Methylation Patterns in Three Rat Models of Acquired Epilepsy. Sci Rep 2016; 6:25668

90. Guo JU, Ma DK, Mo H, et al. Neuronal activity modifies the DNA methylation landscape in the adult brain. Nat. Neurosci. 2011; 14:1345-1351

91. Jagirdar R, Drexel M, Bukovac A, et al. Expression of class II HDACs in two mouse models of temporal lobe epilepsy. J. Neurochem. 2015; 136:717-730

92. Kobow K, Blümcke I. Epigenetic mechanisms in epilepsy. Prog. Brain Res. 2014; 213:279-316

93. Crosio C, Heitz E, Allis CD, et al. Chromatin remodeling and neuronal response: multiple 
signaling pathways induce specific histone $\mathrm{H} 3$ modifications and early gene expression in hippocampal neurons. J. Cell. Sci. 2003; 116:4905-4914

94. Sng JCG, Taniura H, Yoneda Y. Histone modifications in kainate-induced status epilepticus. Eur. J. Neurosci. 2006; 23:1269-1282

95. Nainar S, Marshall PR, Tyler CR, et al. Evolving insights into RNA modifications and their functional diversity in the brain. Nat. Neurosci. 2016; 19:1292-1298

96. Chinthapalli K, Bartolini E, Novy J, et al. Atypical face shape and genomic structural variants in epilepsy. Brain 2012; 135:3101-3114

97. Coppola A, Chinthapalli K, Hammond P, et al. Pediatric diagnosis not made until adulthood: a case of Wolf-Hirschhorn syndrome. Gene 2013; 512:532-535

98. Stern WM, Sander JW, Rothwell JC, et al. Impaired intracortical inhibition demonstrated in vivo in people with Dravet syndrome. Neurology 2017; 88:1659-1665

99. Yu FH, Mantegazza M, Westenbroek RE, et al. Reduced sodium current in GABAergic interneurons in a mouse model of severe myoclonic epilepsy in infancy. Nat. Neurosci. 2006; 9:1142-1149

100. Sun Y, Paşca SP, Portmann T, et al. A deleterious Nav1.1 mutation selectively impairs telencephalic inhibitory neurons derived from Dravet Syndrome patients. Elife 2016; 5:479

101. Stern WM, Desikan M, Hoad D, et al. Spontaneously Fluctuating Motor Cortex Excitability in Alternating Hemiplegia of Childhood: A Transcranial Magnetic Stimulation Study. PLoS ONE 2016; 11:e0151667

102. Zeisel A, Muñoz-Manchado AB, Codeluppi S, et al. Brain structure. Cell types in the mouse cortex and hippocampus revealed by single-cell RNA-seq. Science 2015; 347:1138-1142

103. Tasic B, Menon V, Nguyen TN, et al. Adult mouse cortical cell taxonomy revealed by single cell transcriptomics. Nat. Neurosci. 2016; 19:335-346

104. Jaffer F, Avbersek A, Vavassori R, et al. Faulty cardiac repolarization reserve in alternating hemiplegia of childhood broadens the phenotype. Brain 2015; 138:2859-2874

105. Devinsky O, Cross JH, Laux L, et al. Trial of Cannabidiol for Drug-Resistant Seizures in the Dravet Syndrome. N. Engl. J. Med. 2017; 376:2011-2020

* Double-blind, placebo-controlled trial of cannabidiol for the treatment of drug-resistant seizures in Dravet Syndrome, showing greater reduction in the frequency of convulsive seizures and higher rates of adverse effects with cannabidiol compared with placebo.

106. Hsiao J, Yuan TY, Tsai MS, et al. Upregulation of Haploinsufficient Gene Expression in the Brain by Targeting a Long Non-coding RNA Improves Seizure Phenotype in a Model of Dravet Syndrome. EBioMedicine 2016; 9:257-277

107. Cunningham M, Cho J-H, Leung A, et al. hPSC-derived maturing GABAergic interneurons ameliorate seizures and abnormal behavior in epileptic mice. Cell Stem Cell 2014; 15:559-573 